\title{
HLA class I antigen and transporter associated with antigen processing downregulation in metastatic lesions of head and neck squamous cell carcinoma as a marker of poor prognosis
}

\author{
NOBUYUKI BANDOH ${ }^{1,2}$, TAKESHI OGINO ${ }^{1}$, AKIHIRO KATAYAMA ${ }^{1}$, MIKI TAKAHARA ${ }^{1}$, \\ AKIHIRO KATADA ${ }^{1}$, TATSUYA HAYASHI ${ }^{1}$ and YASUAKI HARABUCHI ${ }^{1}$ \\ ${ }^{1}$ Department of Otolaryngology-Head and Neck Surgery, Asahikawa Medical College, Asahikawa; \\ ${ }^{2}$ Head and Neck Cancer Center, Hokuto Hospital, Obihiro, Japan
}

Received October 23, 2009; Accepted December 14, 2009

DOI: $10.3892 /$ or_00000717

\begin{abstract}
HLA class I antigen processing machinery plays a crucial role in the generation of peptides from endogeneously synthesized proteins and in their presentation to cytotoxic T lymphocytes. The purpose of this study was to analyze the downregulation of HLA class I antigen, transporter associated with antigen processing (TAP) and tapasin in primary and metastatic lesions of head and neck squamous cell carcinoma (HNSCC) and to compare TAP, tapasin and HLA class I antigen downregulation in metastatic lesions with that of primary lesions. We analyzed expression levels of TAP1, TAP2, tapasin and HLA class I antigen in 25 primary and autologous metastatic lesions by staining formalin-fixed, paraffin-embedded tissue sections in the immunoperoxidase reaction. We identified the expression levels of TAP1, TAP2, tapasin and HLA class I antigen were coordinately downregulated in both primary and metastatic lesions and were significantly lower in metastatic lesions than in autologous primary lesions tested. HLA class I antigen downregulation in metastatic lesion was significantly associated with reduced disease-free survival of patients $(\mathrm{P}<0.05)$. Multivariate Cox proportional hazards model analysis identified negativity of HLA class I antigen as an independent prognostic marker. HLA class I antigen and TAP are likely to be downregulated in metastatic lesions compared with primary lesions in HNSCC. The higher frequency of HLA class I antigen and TAP downregulation in metastases play a role in the clinical course of the disease.
\end{abstract}

Correspondence to: Dr Nobuyuki Bandoh, Head and Neck Cancer Center, Hokuto Hospital, Inadacho Kisen 7-5, Obihiro, Hokkaido 080-0833, Japan

E-mail: bando@hokuto7.or.jp

Key words: head and neck squamous cell carcinoma, HLA class I antigen, tapasin, transporter associated with antigen processing

\section{Introduction}

Malignant transformation of human cells is frequently associated with changes in HLA class I antigen expression and/or function (1-4). These changes may affect the interactions of malignant cells with the host's immune system and especially with HLA class I antigen restricted, tumor antigen (TA)-specific CTL. As a result they may have a negative impact on the clinical course of malignant disease as well as on the outcome of $\mathrm{T}$ cell-based immunotherapy (5). Many molecular mechanisms have been shown to underlie defects in HLA class I antigen expression and function. Among them are abnormalities in the HLA class I antigen processing machinery (APM). In previous years the role of this machinery in the generation and stable expression of HLA class I antigens on the cell membrane has been well characterized (6). Peptides presented by HLA class I antigens are generated mostly from intracellular antigens by the proteasome which sharpens its proteolytic activity by replacing the delta (Y), MB1 (X) and Z subunits with low molecular weight protein (LMP)2, LMP7 and LMP10 subunits, respectively, when exposed to interferon (IFN) $-\gamma$ (7). The generated peptides are translocated by the transporter associated with antigen processing (TAP) 1 and TAP2 complex into the endoplasmic reticulum (ER) where the chaperones calnexin, calreticulin and ERp57 are involved in the correct folding and assembly of nascent HLA class I heavy chains with $\beta_{2}$ microglobulin $\left(\beta_{2} \mathrm{~m}\right)(8,9)$. The resulting complexes are bridged by tapasin to TAP which loads peptides. Following dissociation from TAP and ER chaperones, the trimolecular complex travels through the Golgi secretory pathway to the cell surface for presentation of peptides to CTLs (10). Defects in TAP1, TAP2 and tapasin may result in HLA class I antigen loss or downregulation on the cell surface, as indicated by TAP and tapasin-deficient cell lines (11-13).

Head and neck squamous cell carcinoma (HNSCC) is the most common malignancy of the upper aerodigestive tract. Immunohistochemical staining of frozen and/or formalin-fixed, paraffin-embedded HNSCC primary lesions has detected HLA class I antigen loss and downregulation with a frequency ranging from 6 to $81 \%$ (14-21). The mechanisms underlying the HLA class I antigen defects in HNSCC lesions have been analyzed only to a limited extent. In particular scanty 
information is available on the role of TAP1, TAP2 and tapasin defects in the generation of the abnormal HLA class I antigen phenotypes identified in HNSCC lesions. The lack of this information has a negative impact on the design of strategies to correct HLA class I antigen defects in HNSCC lesions. Therefore, in the present study we have analyzed TAP1, TAP2 and tapasin expression in primary and autologous metastatic lesions. We have correlated the results of immunohistochemical staining of the lesions with the expression of HLA class I antigen and with the disease progression.

\section{Patients and methods}

Patients and tissue samples. Tumor specimens from 25 patients included biopsies for diagnostic purposes and primary lesions and metastatic lymph nodes surgically removed for therapeutic purposes. Tissue samples were fixed with $20 \%$ buffered formalin, routinely processed and embedded in paraffin. None of the patients had received preoperative therapy. All the patients signed informed consent for tissue studies, which had received prior approval from the Institutional Review Board.

Monoclonal antibodies. The TAP1-specific mAb NOB-1 and the TAP2-specific mAb NOB-2 are secreted by hybridomas derived from the fusion of murine myeloma cells P3-X63Ag8.653 with splenocytes from BALB/c mice immunized with partial length TAP1 recombinant fusin protein (amino acids 434-735) and a keyhole limpet hemocianin-conjugated TAP1 peptide (amino acids 717-735) and with partial length TAP2 recombinant protein (amino acids 316-703), respectively. The specificity of the mAbs was assessed by their reactivity with molecules with the size corresponding the immunizing TAP1 and TAP2 when tested in Western blotting with a lysate of the human T2 cells and the mouse myeloma cells P3-X63AG8.653 (22). The mAb HC-10 which recognizes a determinant expressed on all $\beta_{2} \mathrm{~m}$-free HLA-B and $\mathrm{C}$ heavy chains and on $\beta_{2}$ m-free HLA-A10, -A28, -A29, -A30, -A31, -A32 and -A33 heavy chains were used (23). The tapasinspecific mAb TO-3 was developed as described (24). The anti-idiotypic mAb MK2-23 used as an isotype-negative control was developed as described (25).

Immunoperoxidase staining of formalin-fixed, paraffinembedded tissue sections. Immunoperoxidase staining of formalin-fixed, paraffin-embedded tissue sections with mAb was performed utilizing the EnVision+ system (Dako Cytomation, Carpinteria, CA, USA) as described (26). The percentage of stained tumor cells in each lesion was evaluated independently by two investigators. Normal lymphocytes and/or vessel endothelia were used in each specimen as internal positive controls. Staining with mAb MK2-23 was used as a negative control. Results were scored as positive (+), heterogeneous $( \pm)$ and negative (-) when the percentage of stained tumor cells in an entire lesion was $(75 \%, 25-75 \%$ and (25\%, respectively, according to the criteria established by the HLA and Cancer component of the 12th International Histocompatibility Workshop (27).

Statistical analysis. Differences of TAP1, TAP2, tapasin and HLA class I antigen expression in primary lesions from those
Table I. Clinicopathological characteristics of the patients with HNSCC included in the study.

\begin{tabular}{|c|c|}
\hline Characteristic & No. of patients \\
\hline Age (years) & $51-77$ \\
\hline \multicolumn{2}{|l|}{ Gender } \\
\hline Male & $20(80 \%)$ \\
\hline Female & $5(20 \%)$ \\
\hline \multicolumn{2}{|l|}{ Tumor site } \\
\hline Oral cavity & $10(40 \%)$ \\
\hline Larynx & $6(24 \%)$ \\
\hline Hypopharynx & $6(24 \%)$ \\
\hline Oropharynx & $3(12 \%)$ \\
\hline \multicolumn{2}{|c|}{ Primary tumor status } \\
\hline $\mathrm{T} 1$ & $2(8 \%)$ \\
\hline $\mathrm{T} 2$ & $12(48 \%)$ \\
\hline $\mathrm{T} 3$ & $8(32 \%)$ \\
\hline $\mathrm{T} 4 \mathrm{a}$ & $3(12 \%)$ \\
\hline \multicolumn{2}{|c|}{ Metastatic nodal status } \\
\hline N1 & $12(48 \%)$ \\
\hline $\mathrm{N} 2 \mathrm{a}$ & $3(12 \%)$ \\
\hline $\mathrm{N} 2 \mathrm{~b}$ & $8(32 \%)$ \\
\hline $\mathrm{N} 2 \mathrm{c}$ & $2(8 \%)$ \\
\hline \multicolumn{2}{|c|}{ Tumor differentiation } \\
\hline Well & $7(28 \%)$ \\
\hline Moderate & $16(64 \%)$ \\
\hline Poor & $2(8 \%)$ \\
\hline Total & 25 \\
\hline
\end{tabular}

in autologous metastatic lesions were tested utilizing Wilcoxon signed rank test. Correlations among the parameters were determined using the Spearman rank correlation coefficient. Disease-free survival (DFS) was calculated using the KaplanMeier method. Time was defined as the period starting from the date of diagnosis to the date of disease relapse or that of last follow-up visit. A log-rank test was used for screening the possible prognostic factors in relation to the patients' survival. A Cox proportional hazards model was used to determine the relationship between DFS and other variables. Statistical tests were based on a level of significance at $\mathrm{P}<0.05$.

\section{Results}

Patients. Between 1995 and 2005, 25 Japanese patients (20 males and 5 females) ranged from 51 to 77 with a median age of 65 years old were treated for HNSCC in the Department of Otolaryngology-Head and Neck Surgery, Asahikawa Medical College Hospital. Clinicopathological characteristics of the patients are shown in Table I. The anatomic site of primary lesions was the oral cavity in $10(40 \%)$ patients, the larynx in $6(24 \%)$, the hypopharynx in $6(24 \%)$, and the oropharynx in $3(12 \%)$. Tumors were well differentiated in $23(37 \%)$ patients, moderately differentiated in $33(52 \%)$, and poorly differentiated in 7 (11\%). According to the Tumor-NodeMetastasis Classification of Malignant Tumors (6th edition), 

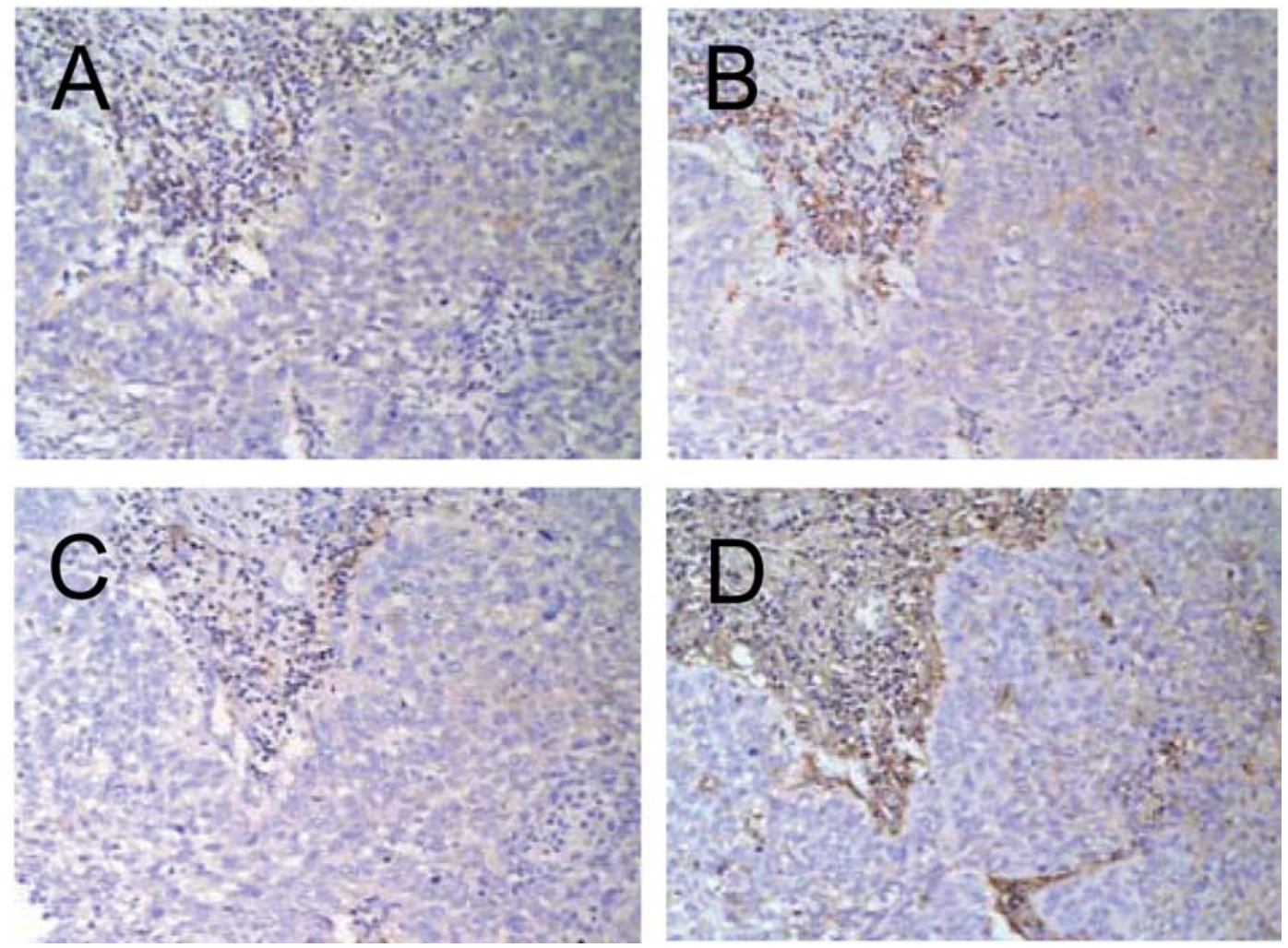

Figure 1. Representative staining patterns for TAP1, TAP2, tapasin and HLA class I antigen of formalin-fixed tissue sections from a primary HNSCC lesion The sections of a primary lesion from patient no. 10 were stained with anti-TAP1 mAb NOB-1 (panel A), anti-TAP2 mAb NOB-2 (panel B), anti-tapasin mAb TO-3 (panel C) and anti-HLA class I heavy chain mAb HC-10 (panel D; x400). Staining of lymphocytes was used as a quality control of the immunohistochemical staining in each tissue section. All the panels were scored as negative.

Table II. TAP1, TAP2, tapasin and HLA class I antigen expression in HNSCC lesions.

\begin{tabular}{|c|c|c|c|c|c|c|c|c|}
\hline \multirow[b]{2}{*}{ Stained tumor cells (\%) } & \multicolumn{2}{|c|}{ TAP1 } & \multicolumn{2}{|c|}{ TAP2 } & \multicolumn{2}{|c|}{ Tapasin } & \multicolumn{2}{|c|}{ HLA class I } \\
\hline & Primary & Metastatic & Primary & Metastatic & Primary & Metastatic & Primary & Metastatic \\
\hline$>75 \%$ (positive) & $8(32 \%)$ & $4(16 \%)$ & $9(36 \%)$ & $3(12 \%)$ & $10(40 \%)$ & $8(32 \%)$ & $9(36 \%)$ & $3(12 \%)$ \\
\hline $25-75 \%$ (heterogeneous) & $15(60 \%)$ & $12(48 \%)$ & $14(56 \%)$ & $16(64 \%)$ & $13(52 \%)$ & $13(52 \%)$ & $13(52 \%)$ & $13(52 \%)$ \\
\hline$<25 \%$ (negative) & $2(8 \%)$ & $9(36 \%)$ & $2(8 \%)$ & $6(24 \%)$ & $2(8 \%)$ & $4(16 \%)$ & $3(12 \%)$ & $9(36 \%)$ \\
\hline
\end{tabular}

the $\mathrm{T}$ classification of the disease was $\mathrm{T} 1$ in $2(8 \%)$ patients, T2 in $12(48 \%), \mathrm{T} 3$ in $8(32 \%)$, and T4a in $3(13 \%)$. the $\mathrm{N}$ classification of the disease at diagnosis was N1 in $12(48 \%)$, $\mathrm{N} 2 \mathrm{a}$ in $3(12 \%), \mathrm{N} 2 \mathrm{~b}$ in $8(32 \%)$, and N2c in $2(8 \%)$ patients.

All the patients were treated with radical surgery composed of local resection for primary tumor and neck dissection for neck node metastasis. Follow-up period of all patients ranged from 2 to 129 months with a median of 61 months. Ten (40\%) of 25 patients died from the disease. The 5-year DFS rate for all patients was $51.2 \%$.

TAP1, TAP2 and tapasin downregulation in relation to HLA class I antigen downregulation in primary HNSCC lesions. To determine whether the downregulation was present in primary HNSCC lesions, TAP1, TAP2, tapasin and HLA class I antigen expression was analyzed in 25 primary lesions by staining formalin-fixed, paraffin-embedded tissue sections with TAP1, TAP2, tapasin and HLA class I heavy chain-specific mAbs.
Fig. 1 shows representative negative staining patterns for TAP1, TAP2, tapasin and HLA class I antigen expression in primary lesion obtained from a patient. As summarized in Table II, TAP1, TAP2, tapasin and HLA class I antigen expression was downregulated (heterogeneous and negative) in $17(68 \%), 16(64 \%), 15(60 \%)$ and $15(60 \%)$ of the 25 primary lesions tested, respectively. Analysis by Spearman rank correlation coefficient showed significant correlations of HLA class I antigen with TAP1 $(\mathrm{P}<0.01)$, TAP2 $(\mathrm{P}<0.01)$, tapasin $(\mathrm{P}<0.05$; Fig. 2).

Comparison of TAP1, TAP2, tapasin and HLA class I antigen expression in metastatic lesions with that in autologous primary lesions in patients with HNSCC. TAP1, TAP2 and HLA class I antigen expression was downregulated in 21 (84\%), $22(88 \%), 17(68 \%)$ and $22(88 \%)$ of the 25 metastatic lesions tested, respectively (Table II). Representative staining pattern of both primary and autologous metastatic lesion is 

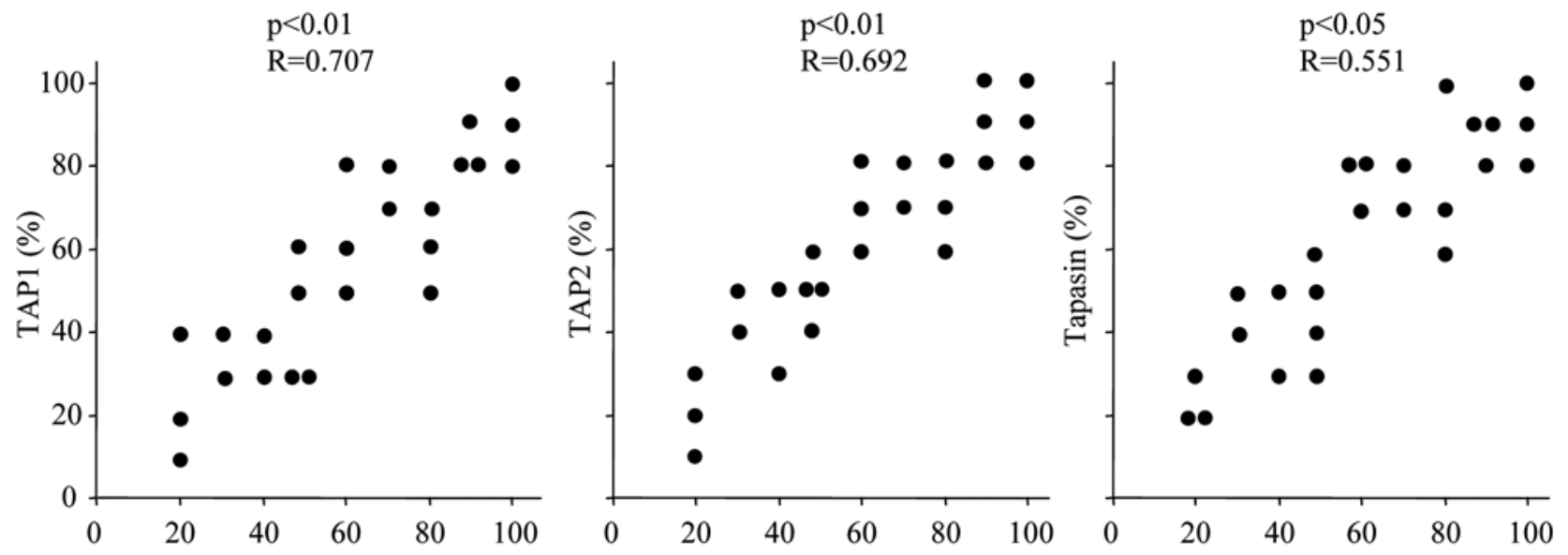

\section{HLA class I antigen (\%)}

Figure 2. Correlation of HLA class I antigen expression with TAP1, TAP2 and tapasin expression in primary HNSCC lesions. Staining by TAP1, TAP2, tapasin and HLA class I heavy chain-specific mAb was evaluated as the percentage of stained tumor cells in each primary lesion. The correlations between the percentage of tumor cells stained by HLA class I heavy chain-specific mAb HC-10 and those of tumor cells stained by TAP1-specific mAb NOB-1, TAP2-specific mAb NOB-2 and tapasin-specific mAb TO-3 in the lesions were analyzed by Spearman rank correlation coefficient.

Table III. Multivariate Cox proportional hazards analysis of DFS for independent prognostic factors in patients with HNSCC.

\begin{tabular}{lccc}
\hline Variable & HR & 95\% CI & P-value \\
\hline Tumor extension (T3 and 4) & 2.63 & $0.62-9.54$ & 0.19 \\
Gender (Male) & 1.24 & $0.34-4.31$ & 0.78 \\
Tumor differentiation (moderate + poor) & 2.01 & $0.55-6.23$ & 0.26 \\
HLA class I antigen (negative) & 4.32 & $1.02-19.53$ & 0.048 \\
\hline
\end{tabular}

HR, hazard ratio; CI, confidence interval.

shown in Fig. 3. Although positive staining of TAP1, TAP2 and HLA class I antigen was seen in the primary lesion, TAP1, TAP2 and HLA class I antigen expression was scored with negative in the autologous metastatic lesion. The expression levels of TAP1, TAP2 and HLA class I antigen were significantly lower in metastatic lesions than in autologous primary lesions tested $(\mathrm{P}<0.05$ each, Fig. 4).

Prognosis according to variables. Kaplan-Meier analysis showed that DFS was significantly worse in patients with positive staining of HLA class I antigen than in patients with negative staining in metastatic lesions $(\mathrm{P}<0.05$; Fig. 5). Significant difference was not identified with regard to TAP1, TAP2 and tapasin expression. To determine whether any of the variables analyzed was an independent prognostic factor, data were analyzed by multivariate Cox proportional hazards model. The variables analyzed include $\mathrm{T}$ classification (T3 and T4), Gender (male), tumor differentiation (moderate + poor differentiation), and HLA class I antigen expression (negative) in metastatic lesions. TAP1, TAP2, and tapasin were excluded from this analysis because each of these variables was correlated with HLA class I antigen expression. Negativity of HLA class I antigen expression in metastatic lesions was the only variable found to be a statistically significant independent prognostic factor for DFS (hazard ratio $(\mathrm{HR})=4.32 ; \mathrm{P}=0.048 ;$ Table III $)$.

\section{Discussion}

Immunohistochemical staining of surgically resected tissue sections from 25 HNSCC patients demonstrated that the expression levels of TAP1, TAP2, tapasin and HLA class I antigen was downregulated in both primary and metastatic lesions. In this study, HLA class I antigen downregulation was $60 \%$ in 25 primary HNSCC lesions. Ferris et al (19) reported that overall defects in HLA class I antigen expression, which range from complete loss to heterogeneous expression, from haplotype loss to selective loss of single allospecificities, has been reported in $60 \%$ of the 491 lesions tested. Our results were consistent with the previous studies.

Among the APM components TAP has been the most extensively investigated in HNSCC lesions, but the results have not been concordant. Immunohistochemical staining of HNSCC lesions has yielded conflicting data on TAP expression. Vora et al (17) reported that TAP1 and TAP2 were downregulated in only $2(9 \%)$ and $0(0 \%)$ of 34 primary HNSCC lesions tested, respectively. Feenstra et al (28) reported that TAP1 and TAP2 were downregulated in 28 

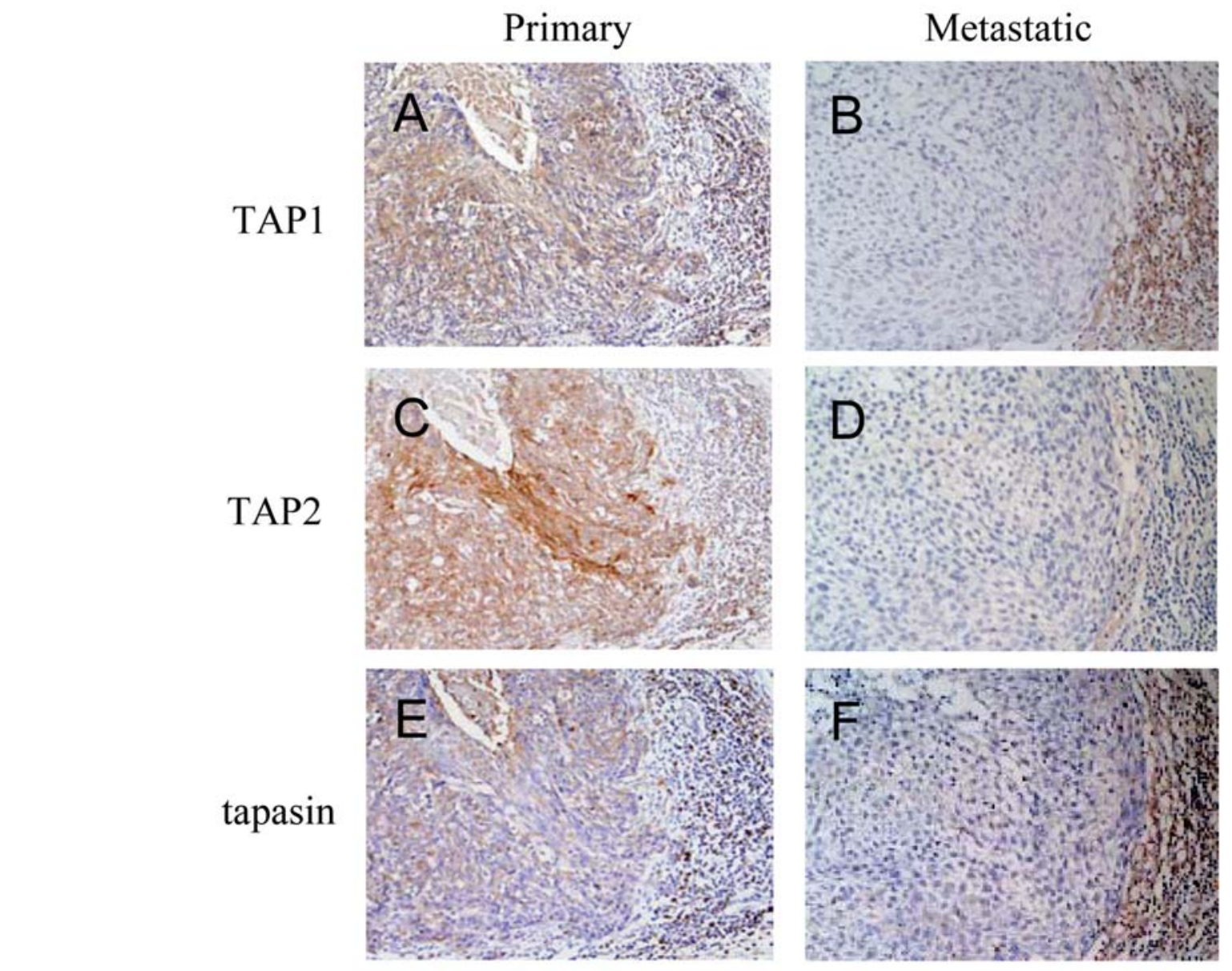

HLA class I antigen
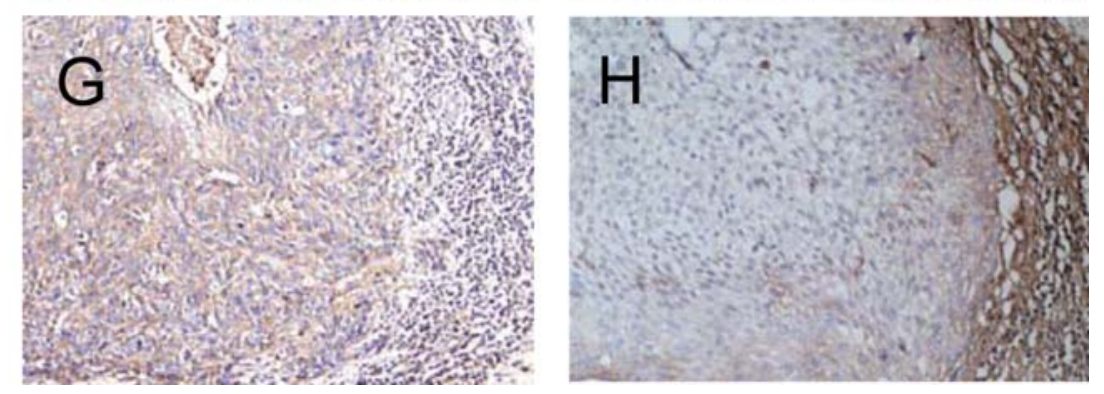

Figure 3. Immunoperoxidase staining for TAP1, TAP2, tapasin and HLA class I antigen of formalin-fixed tissue sections of primary and metastatic HNSCC lesions. The sections of a primary lesion (panels A, C, E and G) and autologous metastatic lesion (panels B, D, F and H) from patient no. 7 were stained with anti-TAP1 mAb NOB-1 (panels A and B), anti-TAP2 mAb NOB-2 (panels C and D), anti-tapasin mAb TO-3 (panels E and F) and anti-HLA class I heavy chain mAb HC-10 (panels G and H; x200). The expression levels of TAP1, TAP2, tapasin and HLA class I antigen in primary lesion were scored as positive. However, the expression levels of TAP1, TAP2, tapasin and HLA class I antigen in autologous metastatic lesion was scored as negative.

$(52 \%)$ and $8(13 \%)$ of 53 primary HNSCC lesions tested, respectively. These results were obtained utilizing frozen tissue sections stained with antiserum. Antisera are difficult to standardize because of differences in antibody content in bleeding samples obtained from an immunized animal and also between immunized animals. Meissner et al reported TAP1 and TAP2 were downregulated in 13 (52\%) and 22 $(88 \%)$ of 25 patients with TAP1-specific mAb 148.3 and TAP2-specific mAb 429.4, respectively (20). TAP1-specific mAb NOB-1 and TAP2-specific mAb NOB-2 are also suitable for staining of formalin-fixed tissue sections. Analysis of staining of formalin-fixed tissue section from cervical carcinoma patients with mAb NOB-1 and NOB-2 showed the frequency of TAP1 and TAP2 downregulation was 23 and $37 \%$, respectively (29). Similarly, negativity of TAP1 and
TAP2 was 33.3 and $26.7 \%$ in patients with ovarian carcinoma with mAb NOB-1 and NOB-2 (30). The association among TAP1, TAP2 and HLA class I antigen expression and their coordinately downregulation in HNSCC in primary lesions suggest that aberrant expression of TAP can be one of mechanisms of tumor escape from immunosurveillance under the circumstances where total loss of HLA class I antigen surface expression is not seen in HNSCC (21).

In this study the expression levels of TAP1, TAP2 and HLA class I antigen were lower in metastatic lesions than in autologous primary lesions in $25 \mathrm{HNSCC}$ lesions tested. The loss of HLA class I antigen expression in primary HNSCC lesions was reported to be associated with regional lymph node metastases (31). It was observed that HLA class I antigen expression was downregulated in $2(22 \%)$ metastatic lesions 

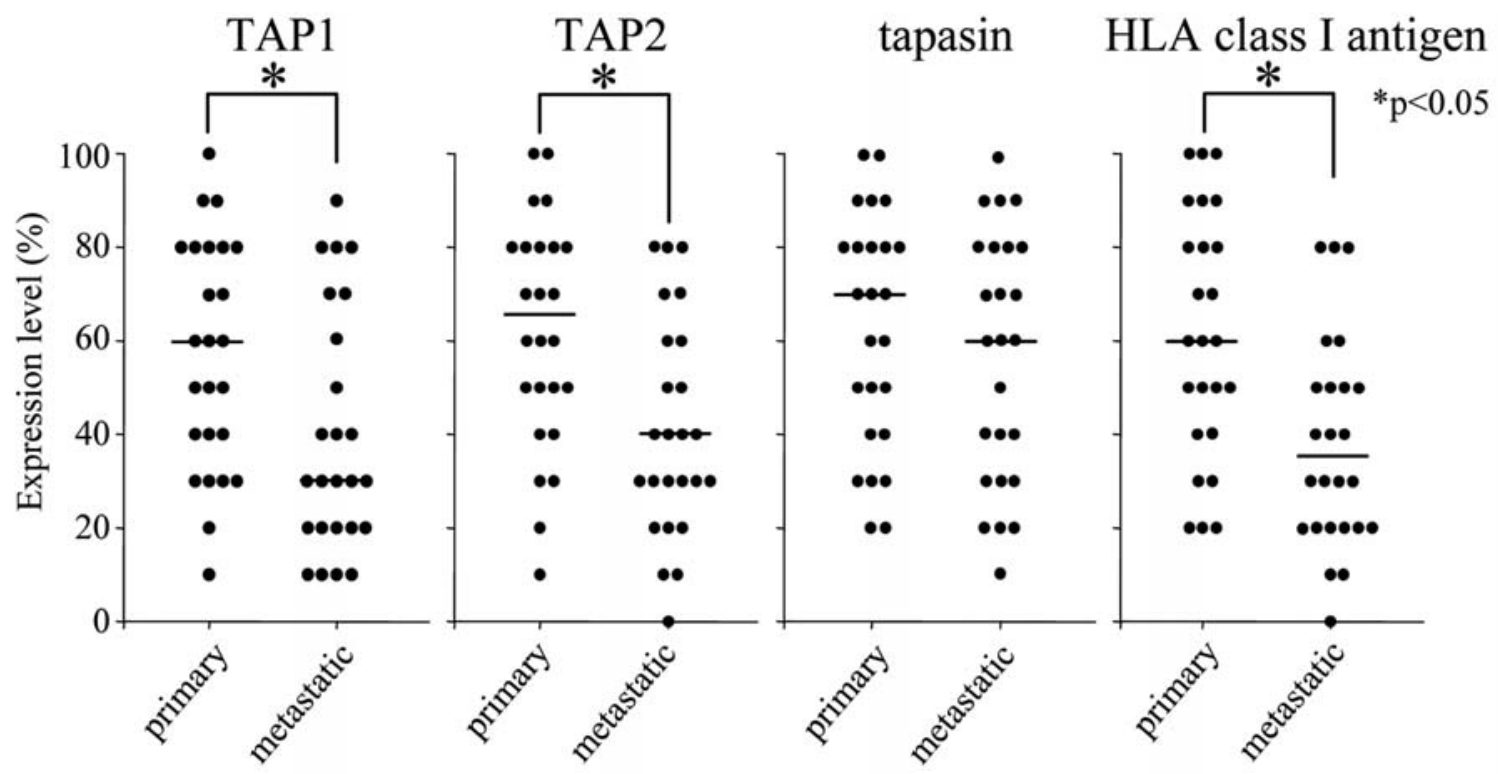

Figure 4. Comparison of TAP1, TAP2, tapasin and HLA class I antigen expression in metastatic lesions with that in autologous primary lesions in patients with HNSCC. Staining with TAP1-specific mAb NOB-1, TAP2-specific mAb NOB-2, tapasin-specific mAb TO-3 and HLA class I heavy chain-specific mAb $\mathrm{HC}-10$ was evaluated as the percentage of tumor cells in each entire lesion. Differences of the expression levels in metastatic lesions from those in primary lesions were analyzed by Wilcoxon signed rank test. The median values are displayed as a short bar (-)

\section{HLA class I antigen}

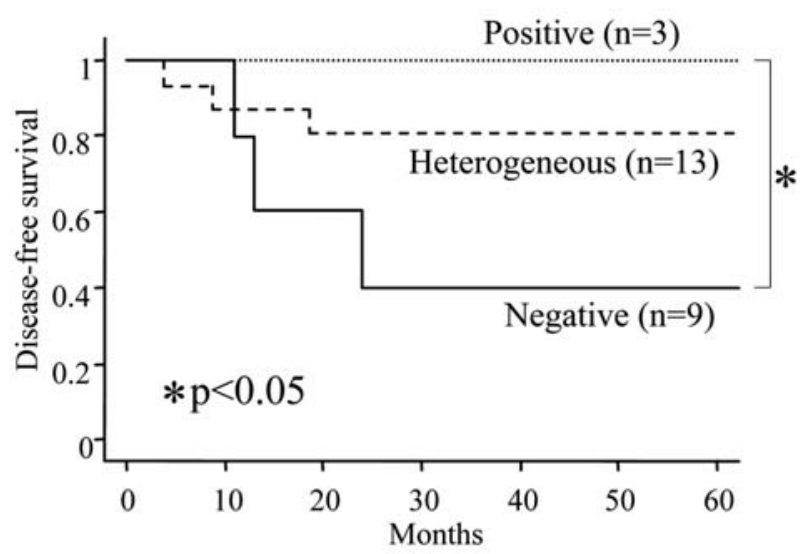

Figure 5. Association of HLA class I antigen downregulation in metastatic HNSCC lesions with disease-free survival (DFS). The DFS of patients with lesions stained with positive (dotted line) and negative (solid line) scores were compared using the Kaplan-Meier method. Differences in patients' survival were analyzed using a log-rank test.

of 9 autologous primary lesions tested (14). However, scanty information is available on the expression of TAP 1 and TAP2 in metastatic HNSCC lesions and about comparison of the expression in primary lesions with that in autologous metastatic lesions. Only one study showed that TAP1 expression was changed to downregulation in 2 (20\%) metastatic lesions when compared with 10 autologous primary lesions tested (14). The frequency of TAP1 downregulation was significantly higher in metastases than in autologous primary lesions in cervical carcinoma (32). In addition TAP1 downregulation was observed in metastases compared with primary lesions in breast (33) and renal cell carcinoma (34).
Our results are compatible with the results as reported in these types of carcinomas. Possible explanation for the coordinate downregulation of HLA class I antigen, TAP1 and TAP2 with the development of metastatic lesions includes immune selection of tumors with an abnormal HLA class I antigen phenotype and/or accumulation of mutations by tumor cells. On the other hand HLA class I antigen downregulation in association with TAP downregulation may possess a selective advantage to metastasize in malignant disease, suggesting an association with tumor aggressiveness.

The association between HLA class I antigen downregulation and poor prognosis has already been described in maxillary sinus SCC (18), laryngeal SCC (21) and esophageal carcinoma (35). Similarly, TAP1, TAP2 and HLA class I antigen downregulation in HNSCC lesions from different anatomic sites was found to be associated with reduced survival (20). In the present study, we showed that HLA class I antigen downregulation in metastatic lesions is significantly correlated with an unfavorable clinical course of the disease in patients with HNSCC. Furthermore, using multivariate analysis, we showed that negativity of HLA class I antigen in metastatic lesions is an independent prognostic factor for disease-free survival. These findings may reflect the lack of recognition by HLA class I antigenrestricted, TA-specific CTL of HNSCC metastatic cells which have lost or down-regulated HLA class I antigen expression. This possibility is supported by the positive correlation between HLA class I antigen expression levels and $\mathrm{CD}^{+} \mathrm{T}$ cell infiltration into the laryngeal SCC lesions (21).

In conclusion, TAP 1 and TAP 2 downregulation in association with at least in part tapasin downregulation may represent one of the mechanisms of HLA class I antigen downregulation on HNSCC lesions. HLA class I antigen downregulation may provide a selective advantage for HNSCC cells during metastasis. Therefore, analysis for APM 
components, especially TAP1 and TAP2, in HNSCC lesions may contribute to optimize the design of T-cell-based immunotherapeutic strategies as well as the selection of patients for this treatment. In addition, TAP gene transfer has to be considered for inclusion in therapies of HNSCC patients and might provide a method for increasing immune response against tumors.

\section{Acknowledgements}

The authors gratefully thank Professor Soldano Ferrone (Department of Immunology and Pathology, University of Pittsburgh Cancer Institute, Pittsburgh, PA) for kindly providing the mAbs and excellent advice. This study was supported by a Grant-in-Aid (No. 20592009) for scientific research from the Ministry of Education, Science, Culture and Technology of Japan.

\section{References}

1. Marincola FM, Jaffee EM, Hicklin DJ and Ferrone S: Escape of human solid tumors from T-cell recognition: molecular mechanisms and functional significance. Adv Immunol 74: 181-273, 2000

2. Seliger B, Cabrera T, Garrido F and Ferrone S: HLA class I antigen abnormalities and immune escape by malignant cells. Semin Cancer Biol 12: 3-13, 2002.

3. Chang CC, Campoli M and Ferrone S: HLA class I antigen expression in malignant cells: why does it not always correlate with CTL-mediated lysis? Curr Opin Immunol 16: 644-650, 2004.

4. Ferrone $S$ and Whiteside TL: Tumor microenvironment and immune escape. Surg Oncol Clin N Am 16: 755-774, viii, 2007.

5. Ferrone S: Immunotherapy dispenses with tumor antigens. Nat Biotechnol 22: 1096-1098, 2004.

6. Lehner PJ, Surman MJ and Cresswell P: Soluble tapasin restores MHC class I expression and function in the tapasin-negative cell line 220. Immunity 8: 221-231, 1998.

7. Fruh K, Gossen M, Wang K, Bujard H, Peterson PA and Yang Y: Displacement of housekeeping proteasome subunits by MHCencoded LMPs: a newly discovered mechanism for modulating the multicatalytic proteinase complex. EMBO J 13: 3236-3244, 1994.

8. Pamer E and Cresswell P: Mechanisms of MHC class I - restricted antigen processing. Annu Rev Immunol 16: 323-358, 1998.

9. Cresswell P, Arunachalam B, Bangia N, et al: Thiol oxidation and reduction in MHC-restricted antigen processing and presentation. Immunol Res 19: 191-200, 1999.

10. Zinkernagel RM and Doherty PC: MHC-restricted cytotoxic T cells: studies on the biological role of polymorphic major transplantation antigens determining T-cell restrictionspecificity, function, and responsiveness. Adv Immunol 27: 151-177, 1979 .

11. Salter RD, Howell DN and Cresswell P: Genes regulating HLA class I antigen expression in T-B lymphoblast hybrids. Immunogenetics 21: 235-246, 1985.

12. Garbi N, Tan P, Diehl AD, et al: Impaired immune responses and altered peptide repertoire in tapasin-deficient mice. Nat Immunol 1: 234-238, 2000 .

13. Gao B, Adhikari R, Howarth M, et al: Assembly and antigenpresenting function of MHC class I molecules in cells lacking the ER chaperone calreticulin. Immunity 16: 99-109, 2002.

14. Esteban F, Concha A, Huelin C, et al: Histocompatibility antigens in primary and metastatic squamous cell carcinoma of the larynx. Int J Cancer 43: 436-442, 1989

15. Houck JR, Sexton FM and Zajdel G: HLA class I and class II antigen expression on squamous cell carcinoma of the head and neck. Arch Otolaryngol Head Neck Surg 116: 1181-1185, 1990.

16. Mattijssen V, De Mulder PH, Schalkwijk L, Manni JJ, Van't Hof-Grootenboer B and Ruiter DJ: HLA antigen expression in routinely processed head and neck squamous cell carcinoma primary lesions of different sites. Int J Cancer (Suppl) 6: 95$100,1991$.
17. Vora AR, Rodgers S, Parker AJ, Start R, Rees RC and Murray AK: An immunohistochemical study of altered immunomodulatory molecule expression in head and neck squamous cell carcinoma. Br J Cancer 76: 836-844, 1997.

18. Ogino T, Bandoh N, Hayashi T, et al: Association of tapasin and HLA class I antigen down-regulation in primary maxillary sinus squamous cell carcinoma lesions with reduced survival of patients. Clin Cancer Res 9: 4043-4051, 2003.

19. Ferris RL, Hunt JL and Ferrone S: Human leukocyte antigen (HLA) class I defects in head and neck cancer: molecular mechanisms and clinical significance. Immunol Res 33: 113-133, 2005.

20. Meissner M, Reichert TE, Kunkel M, et al: Defects in the human leukocyte antigen class I antigen processing machinery in head and neck squamous cell carcinoma: association with clinical outcome. Clin Cancer Res 11: 2552-2560, 2005.

21. Ogino T, Shigyo H, Ishii H, et al: HLA class I antigen downregulation in primary laryngeal squamous cell carcinoma lesions as a poor prognostic marker. Cancer Res 66: 92819289,2006

22. Wang X, Campoli M, Cho HS, et al: A method to generate antigen-specific $\mathrm{mAb}$ capable of staining formalin-fixed, paraffin-embedded tissue sections. J Immunol Methods 299: $139-151,2005$

23. Stam NJ, Spits H and Ploegh HL: Monoclonal antibodies raised against denatured HLA-B locus heavy chains permit biochemical characterization of certain HLA-C locus products. J Immunol 137: 2299-2306, 1986.

24. Ogino T, Wang X, Kato S, Miyokawa N, Harabuchi Y and Ferrone S: Endoplasmic reticulum chaperone-specific monoclonal antibodies for flow cytometry and immunohistochemical staining. Tissue Antigens 62: 385-393, 2003

25. Kusama M, Kageshita T, Chen ZJ and Ferrone S: Characterization of syngeneic antiidiotypic monoclonal antibodies to murine anti-human high molecular weight melanoma-associated antigen monoclonal antibodies. J Immunol 143: 3844-3852, 1989.

26. Bandoh N, Ogino T, Cho HS, et al: Development and characterization of human constitutive proteasome and immunoproteasome subunit-specific monoclonal antibodies. Tissue Antigens 66: 185-194, 2005.

27. Charron D (ed). HLA and Cancer. EDK, Paris, pp445-452, 1997.

28. Feenstra M, Veltkamp M, van Kuik J, et al: HLA class I expression and chromosomal deletions at $6 p$ and $15 q$ in head and neck squamous cell carcinomas. Tissue Antigens 54: 235-245, 1999.

29. Mehta AM, Jordanova ES, Kenter GG, Ferrone S and Fleuren GJ: Association of antigen processing machinery and HLA class I defects with clinicopathological outcome in cervical carcinoma. Cancer Immunol Immunother 57: 197-206, 2008.

30. Han LY, Fletcher MS, Urbauer DL, et al: HLA class I antigen processing machinery component expression and intratumoral T-Cell infiltrate as independent prognostic markers in ovarian carcinoma. Clin Cancer Res 14: 3372-3379, 2008.

31. Grandis JR, Falkner DM, Melhem MF, Gooding WE, Drenning SD and Morel PA: Human leukocyte antigen class I allelic and haplotype loss in squamous cell carcinoma of the head and neck: clinical and immunogenetic consequences. Clin Cancer Res 6: 2794-2802, 2000.

32. Cromme FV, van Bommel PF, Walboomers JM, et al: Differences in MHC and TAP-1 expression in cervical cancer lymph node metastases as compared with the primary tumours. Br J Cancer 69: 1176-1181, 1994

33. Kaklamanis L, Leek R, Koukourakis M, Gatter KC and Harris AL: Loss of transporter in antigen processing 1 transport protein and major histocompatibility complex class I molecules in metastatic versus primary breast cancer. Cancer Res 55: 5191-5194, 1995.

34. Atkins D, Ferrone S, Schmahl GE, Storkel S and Seliger B: Down-regulation of HLA class I antigen processing molecules: an immune escape mechanism of renal cell carcinoma? J Urol 171: 885-889, 2004.

35. Hosch SB, Izbicki JR, Pichlmeier U, et al: Expression and prognostic significance of immunoregulatory molecules in esophageal cancer. Int J Cancer 74: 582-587, 1997. 